\title{
DISKS AROUND T TAURI STARS
}

\author{
ANNEILA SARGENT \\ Division of Physics, Mathematics, \& Astronomy, California Institute of \\ Technology, Pasadena, CA, USA 91125 \\ STEVEN BECKWITH \\ Max Planck Institut für Astronomie, Königstuhl 17, W-6900 Heidelberg, \\ Federal Republic of Germany
}

\begin{abstract}
A variety of evidence suggests that at least $50 \%$ of $\mathrm{T}$ Tauri stars are surrounded by disks of gas and dust. Inferred disk properties are compatible with those generally attributed to the solar nebula, before the formation of planets. Millimeter-wave aperture synthesis mapping of molecular line and dust continuum emission from $T$ Tauri stars provides a direct way to ascertain if disks are present. The technique enables detailed studies of disk temperature and density distributions, chemical properties and velocity fields. These results inform our understanding of both star formation and solar system evolution. We review the observations to date and discuss directions for future research.
\end{abstract}

\section{INTRODUCTION}

Millimeter wavelength interferometry enables us to make detailed measurements of the molecular cloud material that gives rise to stars and remains associated with them for a significant part of their pre-main sequence evolution. The gas and dust around around newly-formed stars is expected to be distributed in disks (c.f. Shu, Adams, \& Lizano 1987; Shu et al. 1992). Observations of early star formation, when an infalling envelope still enshrouds the star-disk system, are described separately in these proceedings by M. Hayashi. Here, we consider the circumstellar disks associated with $\mathrm{T}$ Tauri stars, low-mass, pre-main sequence objects that are already optically visible.

As a class, $T$ Tauri stars have been recognized for nearly 50 years (Joy 1945 ) and their properties are fairly well-established (e.g. Herbig 1962; Bastian et al. 1983; Appenzeller \& Mundt 1989, Bertout 1989). They range in mass from $1-3 M_{\odot}$; their spectra display strong Balmer lines and asymmetric [SII] and [OI] emission line profiles, often with a dominant blue wing; their fluxes at infrared and ultraviolet wavelengths are considerably greater than expected from the stellar photosphere alone; strong polarization is sometimes observed; finally, there is evidence for collimated mass loss in the form of optical jets, molecular outflows, and associated Herbig-Haro objects. Many of these peculiarities are readily accounted for if $\mathrm{T}$ Tauri stars are surrounded by active accretion disks. 
A subset of the class, the "naked" or "weak-line" T Tauri stars, display substantially weaker Balmer lines and much less evidence of disk activity (c.f. Edwards, Ray \& Mundt 1992).

The observed far infrared and millimeter spectral energy distributions (e.g. Adams, Lada \& Shu 1988) provide the most compelling evidence that disks exist around at least $50 \%$ of $\mathrm{T}$ Tauri stars (Strom et al. 1989; Beckwith et al. 1990, hereafter BSCG). The optically thick, far infrared continuum fluxes indicate the disk temperature distribution; by $\lambda \approx 1 \mathrm{~mm}$, particle emission from the disk is largely optically thin and a mass can be estimated (BSCG; Adams, Emerson $\&$ Fuller 1990). The disk properties - sizes of order a few hundred AU, masses about $0.1 M_{\odot}$, and temperatures in the $100 \mathrm{~K}$ range at $1 \mathrm{AU}$ from the star - are similar to those of the solar nebula before the formation of planets (c.f. Beckwith \& Sargent 1992a; Lin \& Papaloizu 1985; Safronov \& Ruzmaikina 1985). Thus, the study of $T$ Tauri disks is likely to enhance our understanding of the origins and evolution of the solar system.

Aperture synthesis mapping at millimeter wavelengths offers a particularly effective way to investigate the disks. The spatial resolution achievable with currently-operating millimeter-wave interferometers, $\sim 1^{\prime \prime}$, corresponds to 140 $\mathrm{AU}$ in the nearest star-forming molecular clouds, sufficient for star/disk studies. At a typical radius of $100 \mathrm{AU}$, the disk temperature can be as low as $15 \mathrm{~K}$. Disk material will therefore radiate preferentially at millimeter wavelengths. Since the low-level rotational transitions of numerous molecules lie in the millimeterwave band, a rich selection of spectral lines are accessible for observation. The morphology of the circumstellar gas, and an independent estimate of disk mass, can be derived from these spectral line measurements. Interferometer maps of the $\mathrm{mm}$-wave continuum emission from circumstellar particles indicate the dust morphology and mass. The temperature and density structure, as well as disk chemistry, can be established through high resolution mapping in different molecular species and isotopes. Molecular line interferometry has the added advantage of enabling investigations of disk velocity structure. Moreover, because aperture synthesis measurements are insensitive to relatively constant, largescale emission, contributions by the remnant parent clouds are expected to be minimal.

\section{OBSERVATIONS}

\section{$\mathrm{CO}$ and ${ }^{13} \mathrm{CO}$ Aperture Synthesis Mapping}

The application of millimeter-wave interferometry to disk studies is illustrated by aperture synthesis observations of a number of nearby $T$ Tauri stars. One of the most-studied objects is HL Tauri, a solar-mass, pre-main sequence star in the Taurus star-forming cloud at $140 \mathrm{pc}$. A variety of phenomena, including well-collimated optical jets, high polarization measure and large infrared excess suggest the presence of a disk (e.g. Mundt, Ray \& Bührke 1988, and references therein). Emission from HL Tau has been detected and mapped in the thermal continuum, and in the $\mathrm{CO}$ and ${ }^{13} \mathrm{CO}(1 \rightarrow 0)$ transitions, near $3 \mathrm{~mm}$ (Beckwith et al. 1986; Sargent \& Beckwith $(1987 ; 1991)$. In the ${ }^{13} \mathrm{CO}$ maps near the systemic velocity, a long, narrow structure, perpendicular to the optical jets, extends to radii of $2000 \mathrm{AU}$. Unresolved ( $R \leq 190 \mathrm{AU})$ continuum emission is centered 
on the star. Higher velocity gas is also concentrated towards this core and comprises the bulk of the mass, approximately $0.1 M_{\odot}$. This value agrees well with the mass derived from the continuum spectral energy distribution (BSCG). It is noteworthy from the point of view of early solar system evolution that the positions of emission maxima vary with velocity in a manner that suggests the gas moves in Keplerian orbits.

The morphology and velocity structure of the material around $T$ Tauri, the prototype of the class, have also been analyzed using $\mathrm{CO}$ and ${ }^{13} \mathrm{CO}$ aperture synthesis maps at both 1.3 and $2.6 \mathrm{~mm}$, but the results are as yet inconclusive (Weintraub,.Masson \& Zuckerman 1989; Ghez, Gorham, \& Sargent 1992, in preparation). However, moderate resolution $\left(6^{\prime \prime}\right){ }^{13} \mathrm{CO}(1 \rightarrow 0)$ maps of another $\mathrm{T}$ Tauri star, DG Tauri, indicate disk-like gas structure, extending to about $2000 \mathrm{AU}$ radius (Sargent \& Beckwith 1989). Again, the elongation is along an axis perpendicular to the outflow axis of an optically-visible jet (Mundt 1988), while continuum emission is unresolved (see also Ohashi et al. 1991). Higher resolution line and continuum mapping at $110 \mathrm{GHz}$ (Sargent \& Beckwith 1993, in preparation) demonstrates that a dust core with $R \leq 150 \mathrm{AU}$ is centered on the star. Figure I is a $4^{\prime \prime}$ resolution map of the ${ }^{13} \mathrm{CO}(1 \rightarrow 0)$ emission from DG Tau within $1.3 \mathrm{~km} \mathrm{~s}^{-1}$ of the systemic velocity, $\sim 7.9 \mathrm{~km} \mathrm{~s}^{-1}$. Gas extends to large radii at these low velocities, but there is also a strong concentration at the stellar position. This concentration becomes more dominant when contributions from higher velocity gas are included, again consistent with Keplerian rotation. Estimates from the integrated ${ }^{13} \mathrm{CO}$ intensity, $11.5 \mathrm{Jy} \mathrm{km} \mathrm{s}^{-1}$, and from the 2.7 $\mathrm{mm}$ continuum flux, $75 \mathrm{mJy}$, imply an approximate mass of $0.04-$ to $0.05 M_{\odot}$, in good agreement with the model-based disk mass (BSCG).

Snapshot interferometer maps of the $\lambda=2.6 \mathrm{~mm}$ continuum emission from several other T Tauri stars, GM Aur, DL Tau, RY Tau, and CI Tau (D. Despois et al., these proceedings) are also consistent with the presence of circumstellar disks but do not provide unambiguous proof of their existence. Conclusive evidence should, in principle, be obtainable from analysis of the velocity structure. Unfortunately, a persistent problem is caused by ambient cloud contributions to molecular line emission near the disk systemic velocity. Large-scale cloud structure is resolved out by interferometers, but the maps demonstrate that there is significant emission from relatively small-scale $\left(\leq 15^{\prime \prime}\right)$ features in the ambient gas. These make it especially difficult to isolate velocity structure pertaining only to the disk.

Detailed calculations of the molecular line emission expected from circumstellar disks can, however, simplify analysis of the observations. Recently, such calculations have been undertaken for disks with properties compatible with those of the pre-solar nebula (Beckwith \& Sargent 1992b; Omodaka, Kitamura \& Kawazoe 1992). A preliminary comparison of the results of the model calculations for a rotating ${ }^{13} \mathrm{CO}$ disk with observations of GM Aurigae show reasonable agreement (c.f. Koerner, Sargent \& Beckwith 1992, in preparation), and encourage us to believe that model-fitting of the line emission will soon allow a straightforward interpretation of the observations. However, very high spatial and spectral resolution are vital to the success of this type of analysis. High resolution may also serve to eliminate additional cloud contributions. In recent ${ }^{13} \mathrm{CO}(2 \rightarrow 1)$ maps of HL Tau at $1^{\prime \prime} .3$ resolution, for example, the confusing contributions of ambient cloud and outflowing gas (c.f. Sargent \& Beckwith 1991) 


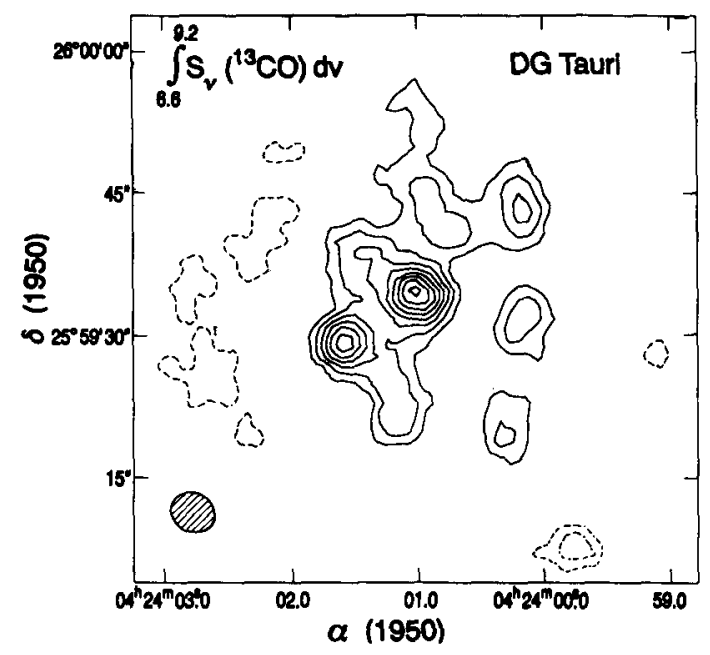

FIGURE I A map of ${ }^{13} \mathrm{CO}$ emission from DG Tau in the velocity range 7.9 $\pm 1.3 \mathrm{~km} \mathrm{~s}^{-1}$. The hatched region represents the $4 . .^{\prime \prime} 5 \times 4 . .^{\prime \prime} 1$ beam. Contours begin at the $2 \sigma$ level, $60 \mathrm{mJy} / \mathrm{beam}$, and are spaced by $30 \mathrm{mJy} / \mathrm{beam}$. The peak flux is $280 \mathrm{mJy} /$ beam, corresponding to a brightness temperature of $1.5 \mathrm{~K}$

are unseen; only a disk-like structure with radius $100 \mathrm{AU}$ is detected (Sargent \& Beckwith 1992, in preparation). Surprisingly, the models calculations demonstrate that even emission in the ${ }^{13} \mathrm{CO}(1 \rightarrow 0)$ transition is optically thick for disk masses $\geq 0.001 M_{\odot}$. Consequently, $\mathrm{CO}$ and ${ }^{13} \mathrm{CO}$ interferometric observations to date have reflected only disk temperature distributions, and have provided no information about the density structure. Studies of disk density variations require maps in rarer isotopes, such as $\mathrm{C}^{18} \mathrm{O}$ or $\mathrm{C}^{17} \mathrm{O}$; these species are much less abundant and emission should be optically thin.

\section{Observations of Other Molecules}

In principle, it should be possible to avoid small-scale ambient cloud contributions through aperture synthesis observations of molecular species that trace only dense gas. Excitation of the CS molecule, for example, requires densities more than an order of magnitude higher that are necessary to stimulate the $\mathrm{CO}$ transitions. Such densities should apply in the disk material closest to $\mathrm{T}$ Tauri stars, but are unlikely to obtain in the ambient clouds.

Interferometeric $\operatorname{CS}(2 \rightarrow 1)$ observations of five $\mathrm{T}$ Tauri stars have recently been reported by Ohashi et al. (1991). Both HL and DG Tau show CS structure that is extended on scales of $1000 \mathrm{AU}$. Remarkably, in neither case is the gas centered on the stars; the displacements, $\approx 3-5^{\prime \prime}$, are significant. No $\operatorname{CS}(2 \rightarrow 1)$ emission was detected from FS Tau, GG Tau or DL Tau, to a $3 \sigma$ limit of $\sim 400$ $\mathrm{mJy} \mathrm{km} \mathrm{s}^{-1}$. Unresolved $98 \mathrm{GHz}$ continuum emission was associated with all the stars except FS Tau, which also went undetected in the BSCG $\lambda=1.3 \mathrm{~mm}$ survey. 
Similar positional offsets were observed by Blake, van Dishoeck \& Sargent (1992) in CS $(2 \rightarrow 1)$ maps of HL Tauri. Combining their maps with single-dish spectra of higher rotational CS transitions, these authors carried out excitation calculations which showed that the conditions in the CS gas around HL Tau are comparable to those in standard cloud cores in the Taurus region. Molecular hydrogen densities are $10^{4}-10^{5} \mathrm{~cm}^{-3}$ and CS fractional abundance about $10^{-8}$. The calculations indicate, moreover, that CS must be depleted by factors of 25 - 50 in any circumstellar disk. Recent mapping of T Tau itself in the $\operatorname{CS}(2 \rightarrow 1)$ transition also shows a dearth of molecular line emission at the stellar position (van Langevelde, van Dishoeck \& Blake, these proceedings).

Such results provide a natural explanation for the positional discrepancies: the CS $(2 \rightarrow 1)$ emission must originate in the ambient core material rather than in the disk. The reasons for CS depletion in T Tauri disks are not yet clear. There may be depletion onto grain mantles; conversion of gas-phase CS into other sulfur-bearing molecules, as in the inner part of the proto-solar nebula (Barshay \& Lewis 1976), is an attractive possibility. It is clearly necessary to search for alternate species that can be employed as density tracers. Initial $\mathrm{HCO}^{+}(1 \rightarrow 0)$ maps of $\mathrm{T}$ Tauri are encouraging in this regard. In contrast to the $\mathrm{CS}$ maps, $\mathrm{HCO}^{+}$emission appears concentrated at the star (H. van Langevelde, E. van Dishoeck \& G. Blake, these proceedings).

\section{Binary T Tauri Stars}

A further complication in unravelling the properties of the material that surrounds $\mathrm{T}$ Tauri stars is introduced by the fact that at least $70 \%$ appear to be members of binary systems (Simon et al. 1992; Leinert et al. 1992; Ghez 1992). Both single-dish and interferometric observations suggest that for systems with orbital periods $\leq 1000$ yrs the disks must be circumbinary; the expected radii are greater than the binary major axis (e.g. Weintraub, Masson \& Zuckerman 1989; BSCG; Beckwith \& Sargent 1992a; Mathieu 1992). High resolution observations of these circumbinary disks are critical to understanding binary formation and evolution. Companion stars may, for example, be produced by global instabilities in the disks (Adams, Ruden \& Shu 1989); loosely bound stars in a cloud may be "captured" through energy dissipation in their disks (Clarke \& Pringle 1992), or cloud fragmentation may result in binaries in a residual disk (Bonnell 1992). The behavior of these star-star systems within disks may also reflect the star-planet interactions in a protosolar nebula.

A recent map of the $2.7 \mathrm{~mm}$ continuum emission in the vicinity of the $\mathrm{T}$ Tauri star, GG Tauri (Simon \& Guilloteau 1992), at 1" resolution, nicely illustrates the differences between the dust distributions around single and multiple stars. GG Tauri is a close $(0 . " 3)$ binary system separated by $10^{\prime \prime}$. from a companion, GG Tau/c, that is also a binary (1."4 separation) (Cohen \& Kuhi 1979; Leinert et al. 1991; Ghez 1992). Unlike the single stars, DG Tau and HL Tau, the $2.6 \mathrm{~mm}$ continuum emission around GG Tau is resolved $\left(3^{\prime \prime} \times 5^{\prime \prime}\right)$ and extended on a scale of several hundred AU towards GG Tau/c. Maps of the 1.3 $\mathrm{mm}$ continuum emission from $\mathrm{T}$ Tau also show a resolved source of radius about $300 \mathrm{AU}$ that surrounds both elements of the binary (Ghez, Gorham \& Sargent 1992 , in preparation), but these results are difficult to interpret since this system emits significant free-free emission (Bieging, Cohen \& Schwartz 1984). 
Maps of the gas emission near the GG Tau - GG Tau/c systems are more tantalizing. At moderate resolution $\left(5^{\prime \prime} \times 3^{\prime \prime}\right)$ the ${ }^{13} \mathrm{CO}(2 \rightarrow 1)$ emission at 1.3 $\mathrm{mm}$ displays a flattened, $3000 \mathrm{AU}$ structure that extends along the GG Tau to GG Tau/c axis and encompasses both binary systems (Koerner, Sargent \& Beckwith 1992). Within this structure, gas appears to be rotating about an east-west axis through GG Tau. However, there is a dramatic change in gas morphology and velocity pattern near GG Tau/c, indicating a distinctly different orientation and rotation axis. Maps covering a larger area, and at higher spatial and spectral resolution, are needed to establish unambiguously the detailed kinematics of this quadruple system.

\section{EXPECTATIONS}

Ongoing expansions and upgrades to the present BIMA, Nobeyama, Plateau de Bure, and Owens Valley arrays, as well as modifications to the VLA that will improve operations at $7 \mathrm{~mm}$, are described elsewhere in these proceedings. The observations presented here summarize the results obtained to date from interferometer studies of $T$ Tauri stars, and indicate the directions we can expect this area of research to take as the current instruments improve in resolution, sensitivity, and mapping efficiency, and as interferometers operating at submillimeter frequencies are constructed.

Improving the rapidity with which detailed maps can be acquired will finally allow surveys of significant numbers of young stars at various stages of early stellar evolution. Searches for $100 \mathrm{AU}$-scale disk morphology and characteristic velocity patterns as a function of stellar properties such as age will become viable. Studies of this type are particularly important to understanding how many stars are likely to support forming solar systems; the time-scales and mechanisms by which the disks dissipate have major implications for planetary system formation.

Increased sensitivity will, of course, enhance these surveys by permitting much deeper searches for direct evidence of disk-structure on $100 \mathrm{AU}$ scales. Disks which display signs of clearing in their inner regions, and therefore are perhaps more evolved, will be of particular interest (c.f. BCSG; Strom, Edwards \& Skrutskie 1992), as will the environments of the weak-line T Tauri stars, where there may be no disks. Greater sensitivity will also enable mapping in less abundant, optically thin species and allow much-needed investigations of disk density structure. With the higher resolution afforded by the expanded arrays, we will begin to examine the disks on the sub-arcsec scales relevant to forming solar system-like planets.

As interferometeric observations at $\lambda=1.3 \mathrm{~mm}$ and at sub-millimeter wavelengths become more commonplace, searches for disk emission in molecules other than $\mathrm{CO}$ should be considerably eased due to the variety of transitions which can be detected and mapped, often simultaneously. Studies of the chemical properties of the disks can be undertaken, with obvious implications for understanding the origin and evolution of the solar system. Although intriguing, interferometric observations of $T$ Tauri stars to date appear to have merely scratched the surface of many problems. In the improved arrays, we will have the tools to seek solutions. 


\section{ACKNOWLEDGMENTS}

The authors are grateful to D. Koerner, G. Blake, E. van Dishoeck, and H. van Langevelde, for communicating their results in advance of publication. A.I.S. thanks NSF for support through Grant AST-9016404 to the Owens Valley array.

\section{REFERENCES}

Adams, F. C., Emerson, J. P., \& Fuller, G. A. 1990, ApJ, 357, 606.

Adams, F.C., Lada, C.J. \& Shu, F.H. 1988, ApJ, 326, 865.

Adams, F.C., Ruden, S.P. \& Shu, F.H. 1989, ApJ, 347, 959.

Appenzeller, I. \& Mundt, R. 1989, A\&A Rev., 1, 291.

Barshay, S.S., \& Lewis, J. S. 1976, ARA $\mathscr{E} A, 14,81$.

Bastian, U., Finkenzeller, U., Jascheck, C., \& Jascheck, M. 1983, $A \& A, 126$ 438.

Beckwith, S. V. W., \& Sargent, A. I. 1992a, in Protostars and Planets III, ed. E. H. Levy, J. I. Lunine \& M. S. Matthews (Tucson:University of Arizona Press), in press.

Beckwith, S. V. W., \& Sargent, A. I. 1992b, ApJ, in press.

Beckwith, S. V. W., Sargent, A. I., Chini, R., \& Güsten, R. 1990, AJ, 99, 924 BSCG.

Beckwith, S., Sargent, A. I., Scoville, N. Z., Masson, C. R., Zuckerman, B., \& Phillips, T. G. 1986, ApJ, 309, 755.

Bertout, C. 1989, ARA\&A, 27, 351.

Bieging, J. H., Cohen, M., \& Schwartz, P. R. 1984, ApJ, 282, 699.

Blake, G. A., van Dishoeck, E. F., \& Sargent, A. I. 1992, ApJ, 391, L99.

Bonnell, I., Martel, H., Bastien, P., Arcoragi, J., \& Benz, W. 1991, ApJ, 377, 553.

Clarke C. J., \& Pringle J. E. 1991, MNRAS, 249, 588.

Cohen, M., \& Kuhi, L. V. 1979, ApJS, 41, 743.

Edwards, S., Ray, T. P., \& Mundt, R. 1992, in Protostars \& Planets III, ed. E. H. Levy, J. I. Lunine, \& M. S. Matthews (Tucson:University of Arizona Press), in press.

Ghez, A. 1992, Ph. D. Thesis, California Institute of Technology.

Herbig, G. H. 1962, $A d v . A B A, 1,147$.

Joy, A. H. 1945, ApJ, $102,168$.

Koerner, D. W., Sargent, A. I., \& Beckwith, S. V. W. ApJ, submitted.

Leinert, Ch. Haas, M., Richichi, A., Zinnecker, H., \& Mundt, R. 1991, $A \mathscr{E} A$, 250, 407.

Leinert, Ch., Weitzel, N., Haas, M., Lenzen, R., Zinnecker, H., Christou, J., Ridgway, S., Jameson, R., \& Richichi, A. 1992, $A \& A$, in press. 
Lin, D.N.C., \& Papaloizou, J. 1985, in Protostars \& Planets II, ed. D. C. Black $\&$ M. S. Matthews (Tuscon: University of Arizona Press), 981.

Mathieu, R.D. 1992, in IAU Symposium No. 151, Interacting Binary Stars, eds. Y. Kondo \& R. Polidan (Dordrecht:Reidel), in press.

Mundt, R. 1988, in NATO-ASI on Formation \& Evolution of Low Mass Stars, eds. A. K. Dupree \& M. T. V. T. Lago (Dodrecht:Kluwer), 257.

Mundt, R., Ray, T.P. \& Bührke, T. 1988, ApJ, 333, L69.

Ohashi, N., Kawabe, R., Hayashi, M., \& Ishiguro, H. 1991, AJ., 102, 2054.

Omodaka, T., Kitamura, Y., \& Kawazoe, E. 1992, ApJ, 396, L87.

Safronov, V. S., \& Ruzmaikina, T. V. 1985, in Protostars B, Planets II, ed. D. C. Black \& M. S. Matthews (Tucson:University of Arizona Press), 959.

Sargent, A. I. \& Beckwith, S. V. W., 1987, ApJ, 323, 294.

Sargent, A. I., \& Beckwith, S. V. W. 1989, in I. A. U. Colloquium No. 120, "Structure and Dynamics of the Interstellar Medium", ed. G. TenorioTagle, J. Melnick, \& M. Moles (Springer-Verlag:Berlin), 221.

Sargent, A. I. \& Beckwith, S. V. W., 1991, ApJ, 382, L31.

Shu, F. H., Adams, F. C., \& Lizano, S. 1987, ARA\&A, 25, 23.

Shu, F. A., Najita, J., Galli, D., \& Ostriker, E. 1992, in Protostars \& Planets III, ed. E. H. Levy, J. I. Lunine, \& M. S. Matthews (Tucson:University of Arizona Press), in press.

Simon, M., Chen, W.P., Howell, R.R., Benson, J.A., \& Slowik, D. 1992, ApJ, 384, 212.

Simon, M., \& Guilloteau, S. 1992, ApJ. 397, L47.

Strom, K. M., Strom, S. E., Edwards, S., Cabrit, S., \& Skrutskie, M. F. 1989, $A J, \boldsymbol{\theta} 7,1451$.

Weintraub, D. A., Masson, C. R., \& Zuckerman, B. 1989, ApJ, 344, 915. 


\section{DISCUSSION}

W.J. Duschl On the one hand, your velocity measurements show very good agreement with Keplerian accretion disks; on the others hand, at the beginning you mentioned that the temperature usually goes $\sim S^{-1 / 2}$ which is not the case in stationary Keplerian disks. Is the exponent $-1 / 2$ mainly due to the inner holes or due to the outer, cooler parts of the disks?

A. Sargent The exponent $-1 / 2$ is derived from the mid-to-far infrared flux distributions as a function of wavelength. These fluxes are dominated by emission from material beyond 100AU from the star. The small value of $q$ is not yet completely understood. It is worth noting that $q$ is correlated with the temperature of the diskhotter disks have the lowest (ie. -0.5 ) values.

R. Kawabe You show the channel maps and velocity map of ${ }^{13} \mathrm{CO}$ emission. The high velocity part of the Keplerian rotation is expected to be concentrated to the central $100 \mathrm{AU}$ scale of the star. But, in you maps the peaks of the CO emission seems to not have peaks at the star position in channel maps. How do you explain?

A. Sargent Perhaps I misunderstand the question - At the systemic velocity of both DG Tau and HL Tau, gas is quite extended. However, since we assume a truncated disk, emission peaks are expected(and found) both close to the star and at distances of $1500 \mathrm{AU}$. At the "high" velocities (systemic $\pm 2 \mathrm{~km} / \mathrm{s}$ here) gas is indeed concentrated at the star. The emission is unresolved in our 2"7 beam(350AU). 